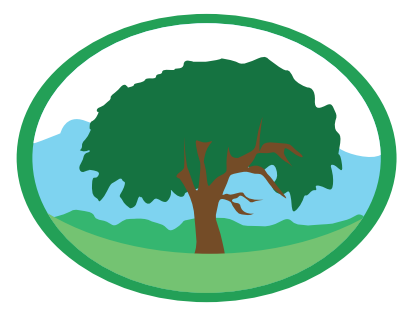

\title{
DIAGNÓSTICO SOBRE A AGRICULTURA DE PRECISÃO NA REGIÃO SUL DO RIO GRANDE DO SUL
}

\author{
VELLAR, R. ${ }^{1}$; GADOTTI, G.I. ${ }^{2}$; LUZ, M.L.G.S² \\ ${ }^{1}$ Eng. Agrícola, Especialista em Biossistemas, retielevellaragmail.com. \\ ${ }^{2}$ Prof. Dr., Centro de Engenharias, Universidade Federal de Pelotas.
}

Palavras-chaves: taxa variável, novas tecnologias, agricultura.

\section{RESUMO}

A Zona Sul do Rio Grande do Sul tem suas bases socioeconômicas tradicionais no setor primário como a soja e o arroz, utiliza a eletrônica embarcada na sua atividade agrícola com o uso de máquinas, sensores, atuadores, softwares e sistemas de informações geográficas via satélites, todos esses com o objetivo de monitorar a operação das máquinas, realizar o controle automático e registrar dados para análise posterior. A Agricultura de Precisão utiliza dessa eletrônica e é uma filosofia de gerenciamento agrícola que busca uma maneira de gerir o campo produtivo metro a metro. Assim, esse trabalho objetivou identificar e diagnosticar a real situação da Agricultura de Precisão na região Sul do Rio Grande do Sul, selecionando aleatoriamente 38 produtores de um universo de 100, para realizar uma pesquisa de opinião com nível de segurança de $95 \%$ de confiança, obtendo como resultado uma nova percepção sobre a Agricultura de Precisão na região em estudo. As conclusões observadas foram o baixo nível da assistência técnica nessa área e o desconhecimento do uso dos equipamentos com a tecnologia de Agricultura de Precisão.

\section{Recognition of precision agriculture in the south of Rio Grande do Sul state, Brazil}

Key-words: variable rate, new technologies, agriculture

\section{ABSTRACT}

The South Zone of Rio Grande do Sul has its traditional socioeconomic bases in the primary sector such as soybean and rice, and uses of electronics on their agricultural activity with the use of machines, sensors, actuators, and geographic information systems software by satellites, with the objective of monitoring the operation of the machines, performing the automatic control and recording data for later analysis. That way it was possible to define the Precision Agriculture(PA) as an agricultural management philosophy that seeks a way to manage a productive field meter by meter. The objective of this study was to identify and to diagnose the real situation of precision farming in the Southern region of Rio Grande do Sul. A number of 38 into 100 producers were randomly selected to answer a poll with confidence level of $95 \%$ that enabled a new perception of the precision agriculture in the region under study. The most relevant findings observed were the level of technical assistance in this area and the unknowing of the use of equipment with PA technology. 


\section{INTRODUÇÃO}

A Zona Sul do Rio Grande do Sul localiza-se no extremo sul do Brasil, uma região de clima subtropical possuindo as quatro estaçóes bem definidas durante o ano. Essa regiáo ocupa uma área de 39.960, 00 $\mathrm{km}^{2}$, apresenta uma população de 853.009 pessoas, distribuídas em 23 municípios: Aceguá, Amaral Ferrador, Arroio do Padre, Arroio Grande, Candiota, Canguçu, Capão do Leão, Cerrito, Chuí, Herval, Jaguarão, Morro Redondo, Pedras Altas, Pedro Osório, Pelotas, Pinheiro Machado, Piratini, Rio Grande, Santa Vitória do Palmar, Santana da Boa Vista, São José do Norte, São Lourenço do Sul e Turuçu (ITEPA, 2011).

A grande maioria das cidades que compóem a Zona Sul tem suas bases socioeconômicas tradicionais no setor primário e secundário, com um parque industrial voltado para transformação de produtos alimentícios como arroz, carne, leite, soja e frutas, bem como um porto exportador, na cidade do Rio Grande, voltado principalmente para commodities agrícolas como soja e arroz (ITEPA, 2011).

É nessas commodities, arroz e soja, que a região se evidencia em relação ao cenário nacional, pois com a demanda crescente da produção de alimentos, a regiâo apresenta todos os recursos naturais disponíveis para elevar seus índices de produção.

A região possui terras, condições climáticas, tecnologia e abundância de água, tudo para ajudar a suprir a carência de alimentos e energias renováveis.

Os desafios contemporâneos relacionados à demanda crescente de alimentos e a necessidade de rendimentos competitivos, além da preservação ambiental, podem encontrar na agricultura de precisão uma eficiente resposta, uma vez que ela é uma ferramenta de gerenciamento e tomada de decisôes que conduz ao aumento de eficiência da utilização dos recursos do sistema produtivo (AMADO; SANTI, 2007).

A agricultura de precisão caracteriza-se pela elevada quantidade de informaçóes disponibilizadas, podendo contribuir para o estabelecimento de relaçóes espaciais de atributos do solo com a produtividade das culturas (AMADO; GIOTTO, 2009). Dessa maneira, a agricultura de precisão procura mudar a forma de gerenciar a produção agrícola, formulando novos conceitos com tecnologias que deixam de ver grandes áreas produtivas como homogêneas, passando a reconhecer sua heterogeneidade, e com essa nova perspectiva é que pode-se definir a Agricultura de Precisão.

A Agricultura de Precisão é um conjunto de técnicas modernas que possibilitam gerenciar a variabilidade espacial e temporal dos atributos do solo, da planta e do ambiente, racionalizando a produção agrícola de maneira que se possa obter maior produtividade como menor custo de insumos e recursos naturais, tornando a atividade mais sustentável e economicamente viável (LUZ, LUZ; GADOTTI, 2013).

A agricultura de precisão também pode ser interpretada como uma filosofia de gerenciamento agrícola que busca informaçóes precisas e se completa com informaçóes exatas, gerenciando o campo produtivo metro a metro, considerando o fato de que cada pedaço da fazenda tem propriedades diferentes (MOLIN, 2002).

Nesse contexto,pode-se explorar o conhecimento detalhado das áreas cultivadas e os fatores de manejo determinantes para a expressão do potencial produtivo de uma cultura.

A eletrônica embarcada na agricultura é representada pelo uso de máquinas agrícolas com sensores, atuadores, softwares e sistemas de informaçóes geográficas via satélites (GPS/GNSS), com o objetivo de monitorar a operação das máquinas, realizar o controle automático e registrar dados para análise posterior. As vantagens do uso da eletrônica embarcada são: melhoria da qualidade da produção, redução das perdas e desgastes, ajuda no planejamento do negócio e proteçâo ao meio ambiente (SARAIVA, 2006).

A introdução da eletrônica embarcada na agricultura brasileira ocorreu de forma gradativa. No período entre 1970 a 1980, a agricultura não contava com benefícios eletrônicos, mesmo porque na época a eletrônica no Brasil era pouco difundida. Praticamente toda forma de manuseio com a terra e suas culturas eram feitas mecanicamente e de forma semiartesanal, com máquinas que ofereciam poucos recursos e alto custo para os usuários(BASLASTREIRE, 2000).

A partir de 1980, surgiram as primeiras máquinas 
com alguma tecnologia e recursos, mas que ainda apresentavam um custo elevado. Havia ainda o paradigma de que uma máquina eletrônica não iria suportar as condiçóes de campo, além da confiabilidade e precisão serem duvidosas. Mas no decorrer da década de 80 o paradigma foi sendo quebrado com melhorias elétricas e mecânicas em relação aos primeiros protótipos de máquinas com módulos eletrônicos (BASLASTREIRE, 2000). Nesta mesma década, na Europa, começaram a ser feitos os primeiros mapas de produtividade e nos EUA ocorreram as primeiras adubaçóes com doses variadas de forma automática(MOLIN, 2002).

Somente a partir de 1990, houve uma explosão do uso da eletrônica na agricultura, com a implantação de sistemas suportando tecnologias cada vez mais confiáveis, com recursos prontos e de fácil utilização, além de apresentar resistência a intempéries e alta precisão. As máquinas sofreram uma considerável queda nos custos, tornando-as acessíveis a pequenos e médios produtores (BASLASTREIRE, 2000) e foi no final dessa década que chegaram ao Brasil as primeiras colhedoras com monitores de produtividade e equipamentos para a aplicaçáo a taxa variada de fertilizantes (MOLIN,2002).

A Agricultura de Precisão passou a existir efetivamente a partir do ano de 2000 quando o governo dos EUA eliminou a degradação do sinal do GPS, que causava um erro exagerado no posicionamento, exigindo um custo muito alto para se ter uma correção diferencial desse sinal. Com essa eliminação, os receptores de navegação de baixo custo se popularizaram e empresas de serviços e consultorias surgiram(MOLIN, 2002).

Também, foi a partir do ano 2000 que as primeiras máquinas brasileiras aplicadoras de defensivos de taxas variáveis de pó e granulados foram fabricadas.

O presente trabalho teve como objetivo identificar e diagnosticar a real situação da Agricultura de Precisão na regiáo Sul do Rio Grande do Sul, além de conhecer seus principais obstáculos e limitaçôes e a partir daí propor soluçóes para que a agricultura da região possa estar à frente da nacional em produtividade, sustentabilidade e viabilidade econômica.

\section{MATERIAL E MÉTODOS}

Primeiramente,foi definido qual tipo de pesquisa seria utilizado para atender a proposta do presente projeto, optando-se por uma "Pesquisa Quantitativa”, pois seus métodos trabalham com indicadores numéricos e seguem critérios estatísticos.

De acordo com as recomendações do SEBRAE (2013), primeiramente é necessário especificar um público-alvo, que, no caso dessa pesquisa foram os produtores rurais, clientes de uma concessionária de máquinas agrícolas,na cidade de Pelotas.Determinado o público-alvo a ser pesquisado, foi estimado o tamanho relativo desse público: clientes que realizaram compras entre o período de janeiro/2011 a junho/2015 na concessionária já citada, totalizando 547 clientes.

Desses 547 clientes selecionou-se a população em estudo, 100 (cem) clientes que adquiriram máquinas com potencial para a agricultura de precisão. Estes foram selecionados, pois compraram equipamentos com potencial para agricultura de precisão no período de janeiro/2011 a junho/2015, e dentre eles foi retirada uma amostra aleatória de38 (pessoas) clientes, conforme SEBRAE (2013).

O grau de homogeneidade dessa população, denominado Split, nesse caso foi classificado como $80 / 20$, esse valor representando um grau de homogeneidade bom, pois a população em estudo possui muitas características em comum onde todas as pessoas possuem o mesmo ramo de atividade (produtores rurais), mesmo sexo, renda similar, etc. representando que o nível de variação das respostas na pesquisa é menor.Portanto,tem-se uma população a ser pesquisada de 100 pessoas com nível de confiança de 95\% e um erro amostral de 10\% (+ou-), Split 80/20, resultando em uma amostra de 38 (pessoas) clientes.

A coleta de dados da pesquisa foi realizada através de saídas a campo onde os clientes eram previamente avisados que seriam entrevistados. Alguns clientes também foram entrevistados pelo telefone devido às condiçóes climáticas muitas vezes não estarem favoráveis para saídas a campo. A época em que foram feitas as entrevistas foi o período de semeadura, um momento crítico para os produtores porque chovia muito e todos estavam atrasados nos trabalhos em suas áreas. 
Os 38 clientes foram entrevistados com perguntas realizadas através de um questionário contendo 13 perguntas objetivas.As perguntas do questionário foram baseadas na metodologia de Sudman; Bradburn(1982). O questionário foi composto por perguntas genéricas, como exemplo: Se o produtor utiliza a Agricultura de Precisão (AP) em sua propriedade? Caso utilize AP, qual a cultura? E a área destinadas AP? Quais maquinários a propriedade dispóe que utilizam algum tipo de tecnologia de AP? Faz amostragem de solo georreferenciada? Com o levantamento dos dados da pesquisa concluído, passou-se para a tabulação dos dados. $\mathrm{Na}$ avaliação dos dados levantados foram considerados: quais as tecnologias encontradas; sua quantificação; se elas são adequadas para a região; em qual cultura é empregada; se possui limitaçôes; se sim, se elas estáo no produtor, no fornecedor, na tecnologia ou na assistência técnica.

As tabulações foram realizadas a partir dos questionários e preenchidas em planilha Excel ${ }^{\circledR}$. Após isso, os dados foram somados e realizadas médias, dependendo do resultado.

\section{RESULTADOS E DISCUSSÃO}

Foram entrevistados 38 clientes residentes na região Sul do Rio Grande do Sul, distribuídos nas cidades de Arroio Grande (6), Cerrito (3), Herval (1), Jaguarão (5), Pedras Altas (3), Pedro Osório (4), Pelotas (3), Piratini (5), Rio Grande (4) e Santa Vitória do Palmar (4) conforme apresentado na Figura 1.

A distribuição geográfica ocorreu aleatoriamente, pois cada saída a campo era condicionada à disponibilidade que a concessionária tinha de deslocar a equipe da regiáo entrevistada, visto que dentro da concessionária existem profissionais específicos para atuar em cada regiáo.

Das culturas de verão semeadas pelos produtores entrevistados destaca-se a soja com um total de 28.535 ha, seguida pelo arroz de total 18.078 ha. Nesse mesmo período, ainda são cultivados milho 370 ha e melancia 560 ha. Como culturas de inverno a pesquisa apresentou como resultado o trigo num total 300 ha e pastagens com total de 7.340 ha. $\mathrm{Na}$ Figura 2estão apresentados a diversidade de culturas semeadas na região, suas médias e somatório total de áreas cultivadas na amostra pesquisada.
Número de entrevistados

38

№ de entrevistados

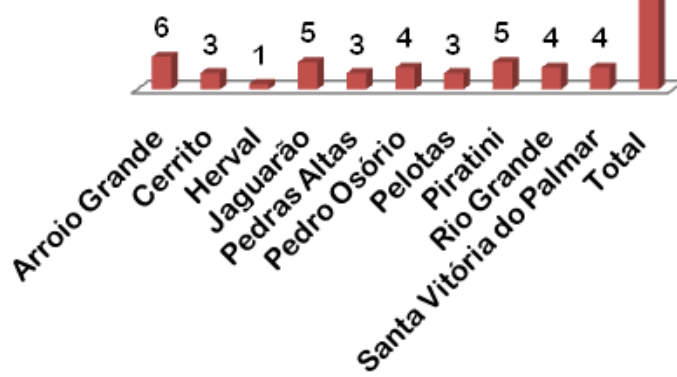

Figura 1 -Distribuição do número de entrevistados em suas respectivas cidades da região Sul do Rio Grande do Sul

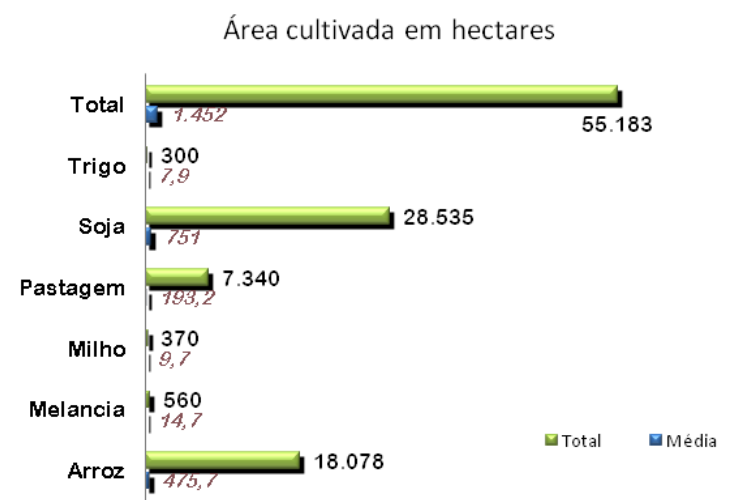

Figura 2 - Área cultivada em hectares de trigo, soja, pastagem, milho, melancia e arroz dos entrevistados pelo estudo

Ao perguntar aos entrevistados "Quando foi iniciado o trabalho de Agricultura de Precisão em sua propriedade?" conforme Tabela 1, observa-se que foi a partir do ano de 2011 que os produtores começaram a destinar algumas áreas da propriedade para a agricultura de precisão, sempre começando pela amostragem de solo georreferenciada em pequenas áreas, muitas experimentais e que não tiveram continuidade.

Tabela 1 - Número de produtores e área aplicada com Agricultura de Precisão (em ha)

\begin{tabular}{|l|l|l|}
\hline Ano & No de produtores & Áreas somadas \\
\hline 2011 & 3 & 929 \\
\hline 2012 & 0 & 0 \\
\hline 2013 & 0 & 0 \\
\hline 2014 & 3 & 740 \\
\hline 2015 & 3 & 2120 \\
\hline Total & 9 & 3789 \\
\hline
\end{tabular}


Do total de entrevistados que iniciaram Agricultura de Precisão pela amostragem georreferenciada apenas $22 \%$ executaram essa atividade, os outros $78 \%$ foram realizados por empresas terceirizadas, cujos nomes e percentuais podem ser observados na Figura 3.

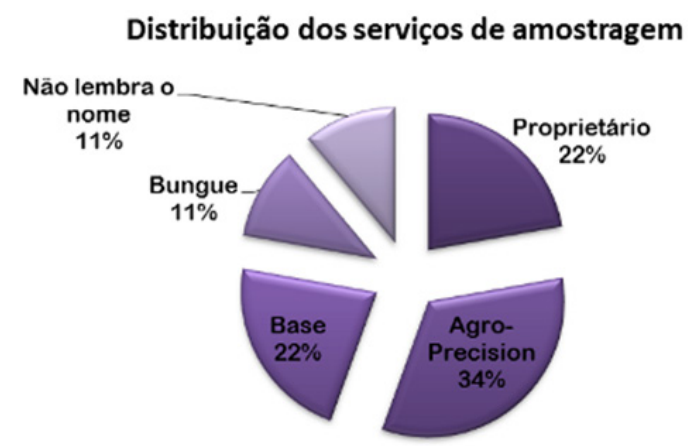

Figura 3 - Distribuição do serviço de amostragem georreferenciada em percentual (\%)

A agricultura de precisão engloba muitos procedimentos específicos, por exemplo, o uso de máquinas agrícolas com recursos eletrônicos (eletrônica embarcada) e de informática como, sensores, atuadores, controladores de pulverização, controladores de adubação, mapeamento e aplicação via satélite.

A Figura 4demonstra que a utilização de sensores nas colhedoras de grãos para confecção de mapas de produtividade metro a metro na lavoura ainda náo é uma prática usual nessa região. Embora exista a tecnologia nas máquinas de alguns produtores, ela não é utilizada. As antenas RTK utilizadas para criar as curvas de nível ou drenos são procedimentos que poderiam estar sendo aplicados mais comumente devido ao grande interesse em especial dos produtores de arroz, porém do total de 55.183ha, somente 970 ha estão utilizando essa tecnologia.

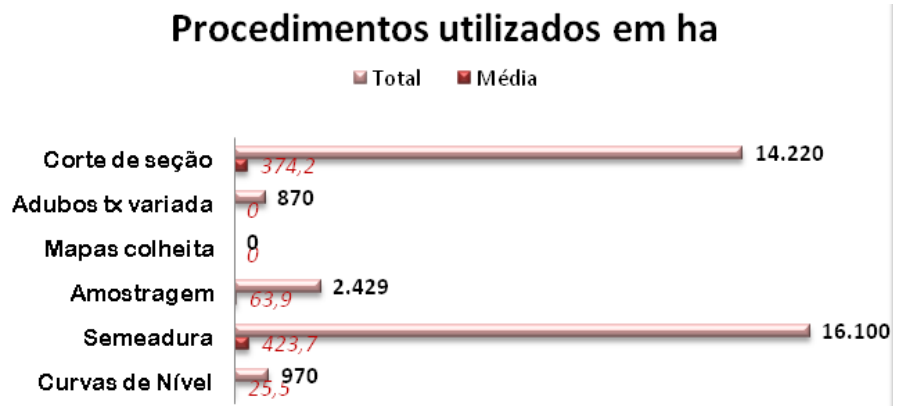

Figura 4 - Número de hectares que utilizam procedimentos de agricultura de precisão no Sul do Rio Grande do Sul
O controle de semeadura com sensores nas linhas das semeadoras que possibilitam o controle de sementes e adubo são utilizadas por uma grande maioria de produtores, totalizando 16.100 ha, mas não possuem taxa variável de sementes.

Nos pulverizadores, os recursos não são utilizados em sua totalidade, onde nenhum dos entrevistados utiliza a aplicação de herbicida ou pesticida com taxas variadas, mas em 14.220 ha são utilizados corte de seção, sendo este parte dos recursos oferecidos nos pulverizadores modernos. A amostragem do solo georreferenciada somou 2.429 ha, porém a aplicação de adubos com taxas variadas que seria o passo seguinte na gestão de Agricultura de Precisão é pouco utilizada, em apenas 870 ha.

A grande maioria dos produtores possui maquinários suficientes e de boa qualidade para a realização do trabalho adequado na lavoura. A Figura 5 apresenta os resultados em relaçáo às quantidades de máquinas equipadas com Agricultura de Precisão que esses produtores possuem até o presente momento. Conforme observado, o uso de pulverizadores com corte de seção é a tecnologia mais adquirida pelos produtores, com $42 \%$, depois segue o piloto elétrico ou hidráulico com $37 \%$, as semeadoras com sensores ocupam $14 \%$, as calcariadoras e antenas RTK apenas 3\%, colhedoras somente $2 \%$ dos entrevistados adquiriram, porém não fazem os mapas de produtividade.

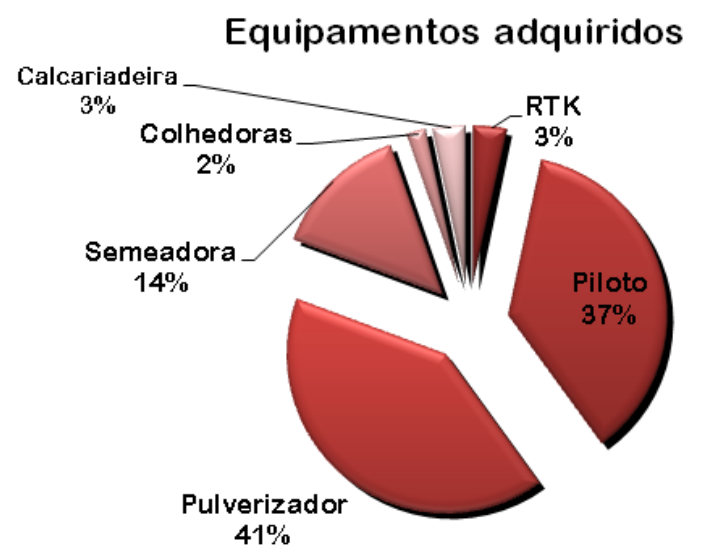

Figura 5 - Equipamentos com Agricultura de Precisão nos produtores entrevistados no Sul do Rio Grande do Sul

Em relação a esses equipamentos adquiridos pelos produtores, foi perguntado: "No momento da entrega na propriedade ocorreu alguma entrega técnica?" e surpreendentemente $41 \%$ responderam que não 
tiveram entrega técnica contra os $59 \%$ que responderam sim. Os percentuais encontrados despertam um sinal vermelho de como essas informaçóes sobre uma nova tecnologia estão sendo transmitidas para os usuários e em que momento ela está sendo transmitida.

$\mathrm{Na}$ Figura 6, foi visualizada uma análise dos clientes, sobre a pós-venda ou assistência técnica desses equipamentos. Apenas 3\% dos entrevistados responderam que a assistência técnica desses equipamentos foi ótima e nesse caso eles estavam se referindo a empresas que prestam assistência em serviços de agricultura de precisão. Para $28 \%$ dos produtores, a assistência foi boa e para outros $28 \%$ a assistência foi ruim. De maneira geral, a maioria dos entrevistados (41\%) considera a assistência como mediana.

\section{Como foi a assistência técnica?}

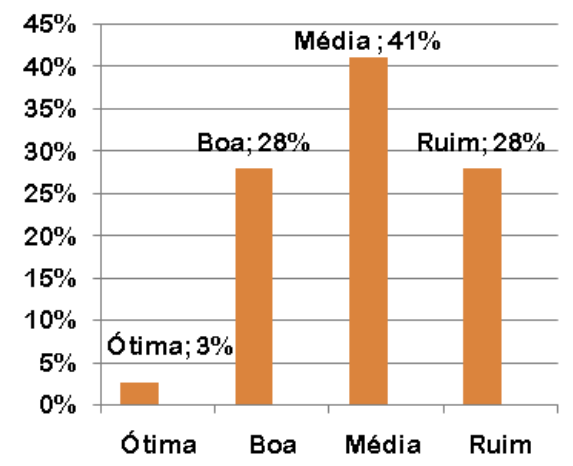

Figura 6 - Classificação do atendimento pós-venda em Agricultura de Precisão no Sul do Rio Grande do Sul

Quando foi perguntado se a Agricultura de Precisão (AP) estava auxiliando os produtores em alguma tomada de decisão em relação ao processo produtivo da propriedade, $74 \%$ dos entrevistados responderam não (ou seja, não percebem a $\mathrm{AP}$ como ferramenta de gestão) e apenas 26\% conseguem tomar alguma decisão com base nos dados fornecidos pela tecnologia.

No final da entrevista, foi perguntado para os produtores o que eles esperam da agricultura de precisão para os próximos anos, e essas respostas foram as mais variadas possíveis. Pôde ser notado, que muitos ainda não estão bem entrosados com a tecnologia, faltando informações sobre seus próprios equipamentos, outros pretendem aderir à tecnologia no futuro. Já, alguns falaram que a tecnologia precisa trazer bons resultados e da importância da relaçáo custo x benefício, proporcionada por tal.

Por último, perguntados se gostariam que as colhedoras de arroz possuíssem essa tecnologia, pois atualmente só as de soja possuem, 55\% não visualizam como uma vantagem ou necessidade para essa cultura.

Assim como foi visto, o agronegócio possui participação fundamental no cenário econômico regional, com reflexos importantes sobre o produto interno bruto, exportaçôes e geração de empregos. Se observada a viabilidade econômica do setor agropecuário a partir da redução dos custos de produção, do aumento da produtividade e da reduçáo do impacto ambiental causado pelo excesso de defensivos agrícolas e combustíveis fósseis, pode-se ressaltar que essa também depende da agricultura de precisão.

Evidencia-se, portanto, que o objetivo da agricultura de precisão vincula-se à maximização na eficiência no uso das máquinas e insumos a partir da tecnologia embarcada e esses devem proporcionar ao produtor informaçóes precisas e exatas de seu sistema produtivo de maneira mais rápida e eficaz para uma melhor gestão.

A pesquisa desenvolvida possibilitou perceber que a assistência da regiáo é classificada como mediana e precisa evoluir em muitos aspectos, como maior agilidade no atendimento e plantôes nos finais de semana.

De maneira geral, é necessário um maior entendimento sobre a Agricultura de Precisão em todos os seguimentos desde o processo, o vendedor até o operador, incluindo também o gestor do agronegócio. Este processo deve começar pelo pleno funcionamento de seus equipamentos, pois quase em sua totalidade os equipamentos adquiridos pelos entrevistados estão sendo subutilizados.

A agricultura de precisão no Sul do Rio Grande do Sul teve inicio em 2011 com amostragem de solo georreferenciada nas lavouras de soja. As colhedoras em sua totalidade não possuem monitor de rendimento e nem realizam mapas de produtividade para avaliar o resultado da produção. Há uma tendência de grande uso de antenas RTK para a construção de curvas de nível para lavouras de arroz e drenos nas áreas de soja. 
Grande parte dos produtores possui pulverizadores autopropelidos com recursos de aplicação de defensivos a taxas variadas, porém somente utilizam o corte de seção.

Por fim, os produtores possuem equipamentos com muitas tecnologias, porém não conseguem utilizá-los em sua totalidade de maneira que essas tecnologias possam auxiliar para uma melhor gestão da sua produçáo,devido a uma existente lacuna de capacitaçáo dos mesmos e seus colaboradores na área de Agricultura de Precisão.

\section{CONCLUSÃO}

As conclusões observadas foram o baixo nível da assistência técnica nessa área e o desconhecimento do uso dos equipamentos com a tecnologia de Agricultura de Precisão.

\section{REFERÊNCIAS}

AMADO, T.J.C.; SANTI, A.L. Agricultura de precisão aplicada ao aprimoramento do manejo do solo. In: FIORIN, J.E., (ed.) Manejo da fertilidade do solo no sistema de plantio direto. Passo Fundo:Berthier, 2007. p.99-144.

AMADO, T.J.C.; GIOTTO, E. A sua lavoura na tela.Revista A Granja, São Paulo, p.38-42, 2009.
BALASTREIRE, L.A. O estado da arte da Agricultura de Precisão no Brasil. Piracicaba, 2000. 224p.

DAMPNEY, P.M.R.; MOORE, M. Precision agriculture in England: current practice and research-based advice to farmers. In: INTERNATIONAL CONFERENCE ON PRECISION AGRICULTURE, 1998, St. Paul. Proceedings... Madison: American Society of Agronomy, 1999. p.661-673.

ITEPA. Banco de dados da zona sul. 2011. Disponível em: $<$ http://www.bancodedadoszonasul.com.br/content>. Acesso em: 10 mar. 2015.

LUZ, M.L.G.S.; LUZ, C.A.S.; GADOTTI, G.I. Agricultura de precisáo.Pelotas: Editora e Gráfica Universitária, 2013, 268p.

MILANI, L.; SOUZA, E.G.; URIBE-OPAZO, M.A.; GABRIEL FILHO, A.; JOHANN, J.A.; PEREIRA, J.O. Unidades de manejo a partir de dados de produtividade.Acta ScientiarumAgronomy, Maringá, v.28, p.591-598, 2006.

MOLIN, J.P. Agricultura de precisáo: o gerenciamento da variabilidade. Piracicaba: ESALQ/USP, 2001, 90p.

MOLIN, J.P. Definição de unidades de manejo a partir de mapas de produtividade.Engenharia Agrícola, Viçosa, v.22, p.83-92, 2002.

SARAIVA, A.M. Eletrônica embarcada e ISOBUS.In: CONGRESSO BRASILEIRO DE AGRICULTURA DE PRECISÃO, 1., 2006. Anais... São Pedro, p.4-5.

SEBRAE, Serviço de apoio às micro e pequenas empresas de Minas Gerais. 3.ed. Belo Horizonte, 2013. Disponível em: <http://www.sebraemg.com.br>. Acesso em: 12 nov. 2015.

SUDMAN, S.; BRADBURN, N. M. Asking questions: a practical guide to questionnaire design.San Francisco: JosseyBass Publishers, 1982. 397p. 